\title{
Assembly-Line-Compatible Electromagnetic Characterization of Antenna Substrates for Wearable Applications using Polynomial Chaos
}

\author{
Thomas Deckmyn \\ INTEC - IDLab \\ Ghent University - imec \\ Ghent, Belgium \\ thomas.deckmyn@ugent.be
}

\author{
Marco Rossi \\ R3S Department \\ Fraunhofer IZM \\ Berlin, Germany \\ marco.rossi@izm.fraunhofer.de
}

\author{
Sam Agneessens \\ INTEC - IDLab \\ Ghent University - imec \\ Ghent, Belgium \\ sam.agneessens@ugent.be
}

\author{
Hendrik Rogier \\ INTEC - IDLab \\ Ghent University - imec \\ Ghent, Belgium \\ hendrik.rogier@ugent.be
}

\author{
Dries Vande Ginste \\ INTEC - IDLAb \\ Ghent University - imec \\ Ghent, Belgium \\ dries.vandeginste@ugent.be
}

\begin{abstract}
For the design and production of reliable devices applied in wearable components, characterization of the electromagnetic properties of materials is of paramount importance. Therefore, we propose a novel approach, based on a resonance-perturbation method, which compares simulations and performed measurements. An inset-fed patch antenna with a resonance frequency in the vicinity of the $2.45-\mathrm{GHz}$ Industrial, Scientific, and Medical band enables us to quickly estimate the characteristics of a given material sample. In a first step, the two frequencies for which the simulated return loss of the fixture crosses a defined threshold are modeled as polynomial functions of the relative permittivity and loss tangent of the material under test. Then, the electromagnetic properties of the material are obtained by comparing the modeled and measured frequencies. The electromagnetic properties of several textile materials of interest are determined with this method. It is shown that the proposed technique is fast, precise, and nondestructive. Owing to this, it is suitable for integration into an assembly line, where substrate samples are straightforwardly characterized before being used for the manufacturing of actual antennas.

Keywords-Resonance perturbation method, substrate
\end{abstract} material characterization, textile antenna.

\section{INTRODUCTION}

Recently, the potential of smart textiles has been unearthed by research on wearable technologies. This has led to a true myriad of novel applications for integrated on-body systems [1], [2]. Such components for wearable applications are usually realized on common, low-cost textiles serving as substrates, while electrotextiles are exploited for the conductive parts. These components are usually lightweight, invisible, flexible and easily integratable into everyday clothing. However, the electromagnetic properties of textile materials, being loss tangent and permittivity, are not widely available. Additionally, they tend to be more inhomogeneous than conventional highfrequency laminates [4]. Taking all of the above into account, it is clear that an estimation of the electromagnetic properties of substrate materials is paramount for designers of wearable components. This claim is amplified by the impending industrialization of textile antenna production, caused by the widespread diffusion of wearable systems within the "Internet of Things". As a result, a fast and reliable characterization technique is needed to cope with the production rate of an assembly line.

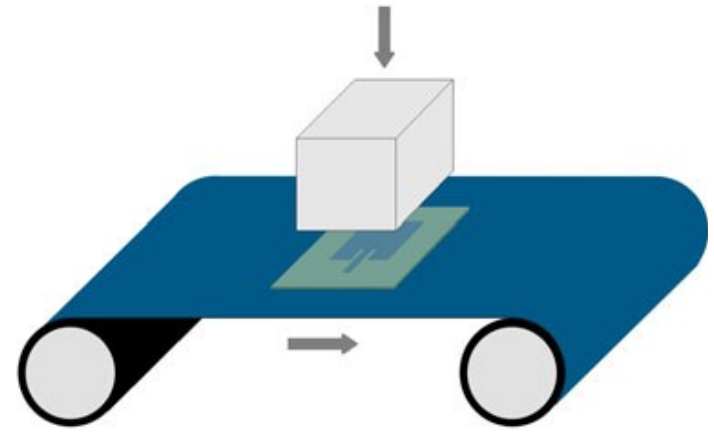

Fig. 1. Assembly line with the MUT unrolled on top of the inset-fed patch antenna for characterization.

The properties of electrotextiles have been extensively investigated in literature [3]. Both non-resonating and resonating methods were proposed for substrate materials. Nonresonating methods exploit broadband transmission lines to extract the material's permittivity and loss tangent from scattering parameter measurements of two textile microstrip lines of different length [5]. Resonant techniques, including planar antenna resonator techniques, are simple to implement, and more accurate and sensitive than non-resonating methods [6], [7]. Yet, all these techniques are usually time consuming and destructive, meaning that the sample cannot be reused after its properties have been determined. Since electromagnetic characteristics of a non-uniform material under test (MUT), such as off-the-shelf textiles, may significantly differ from sample to sample, one sample's characteristics cannot be assumed valid for another one. To overcome these limitations, Rossi et al. [8] proposed a nondestructive resonating technique. However, that measurement fixture is complex, and each fullwave simulation of its resonance frequency takes up to $10 \mathrm{~min}$.

Therefore, we outline a novel efficient nondestructive technique for integration into an industrial production process. First, the MUT is deployed on an inset-fed patch antenna the return loss $\left|S_{11}\right|_{\mathrm{dB}}$ is measured. Next, by comparing simulated and measured resonance frequencies, the variation interval of the relative permittivity $\varepsilon_{\mathrm{r}}$ is found. The variation interval of the loss tangent $\tan \delta$ is set a priori based on practical considerations. For these variation intervals, we model the frequencies at which $\left|S_{11}\right|_{\mathrm{dB}}$ near the resonance peak of the 
fixture crosses a given threshold, as a polynomial function of $\varepsilon_{\mathrm{r}}$ and $\tan \delta$. Finally, the comparison between modeled and measured frequencies yields the MUT's electromagnetic properties. Given the simple measurement fixture and the few full-wave simulations required to estimate the variation interval of $\varepsilon_{\mathrm{r}}$, the construction of the model for a given material only takes a few minutes. Once these models are available, the extraction of $\varepsilon_{\mathrm{r}}$ and $\tan \delta$ is carried out at negligible computational cost for several samples of the same material. The proposed technique is applicable to thin substrates for $\varepsilon_{r}$ up to 10 and $\tan \delta$ up to 0.1 , and for frequencies below $10 \mathrm{GHz}$.

\section{Characterization Procedure}

As applied in an assembly line (see Fig. 1), during the characterization procedure, the MUT is unrolled on top of an inset-fed patch antenna (see Fig. 2). For each sample, the assembly line stops, and a Styrofoam block presses the substrate onto the patch antenna to avoid air gaps without interfering with the return loss $\left|S_{11}\right|_{\mathrm{dB}}$ measurement of the fixture.

The interaction between antenna and MUT shifts the resonance frequency $f_{r}$. We now estimate the properties of the MUT based on this shift, as it depends on the electromagnetic characteristics of the material. First, the variation interval of the relative permittivity $\Delta \varepsilon_{r}$ of the MUT is determined. To this end, we capitalize on the independence of the resonance frequency of the antenna from variations in the MUT's $\tan \delta$. This holds for low-loss materials, such as typical textile substrates applied in antenna design [9]-[11]. We first simulate the structure for an MUT with a permittivity $\varepsilon_{r}^{\text {sim }}$ that yields a higher simulated resonance frequency $f_{r}^{\text {sim }}$ than the measured one $f_{r}^{\text {meas }}$. Specifically, for unknown electromagnetic characteristics of the MUT, as for textile substrates, the initial value of $\varepsilon_{r}^{\text {sim }}$ is set to 1 . In contrast, when properties of the MUT are known or provided by the manufacturer, $\varepsilon_{r}^{\text {sim }}$ is chosen as a lower bound of the known value. Next, we increase $\varepsilon_{r}^{\text {sim }}$ by $\Delta \varepsilon_{r}^{\text {sim }}$ until the simulations yield an $f_{r}^{\text {sim }}$ lower than $f_{r}^{\text {meas }}$. In these simulations, the loss tangent $\tan \delta^{\operatorname{sim}}$ of the MUT remains fixed and can be chosen arbitrarily small. The variation interval of $\varepsilon_{r}$ is finally defined by

$$
\Delta \varepsilon_{r}=\left[\max \left(\varepsilon_{r}^{\operatorname{sim}}-2 \Delta \varepsilon_{r}^{\operatorname{sim}}, 1\right), \varepsilon_{r}^{\operatorname{sim}}+2 \Delta \varepsilon_{r}^{\operatorname{sim}}\right]
$$

with $\varepsilon_{r}^{\text {sim }}$ the last simulated permittivity value. $\Delta \varepsilon_{r}^{\operatorname{sim}}$ is chosen large enough such that $\Delta \varepsilon_{r}$ accounts for non-uniformity of the MUT, determined by only a few simulations. In all cases, $\Delta \varepsilon_{r}^{\text {sim }}=0.1$ has proved to be a suitable choice.

Next, the algorithm extracts the electromagnetic properties of the MUT. First, since all textile materials considered in previous works exhibit a loss tangent tan $\delta$ lower than 0.04 [9]-[11], we set $\Delta \tan \delta$ to $[0,0.1]$. Second, we set a threshold value $t_{\mathrm{dB}}$ to half the peak magnitude of the measured $\left|S_{11}\right|_{\mathrm{dB}}$. This choice yields stable and accurate material properties. Then, we model the two frequencies $f_{\text {sim }}^{(1)}$ and $f_{\text {sim }}^{(2)}$ at which the antenna's simulated return loss $\left|S_{11}\right|_{\mathrm{dB}}$ crosses $t_{\mathrm{dB}}$ as functions of $\varepsilon_{r}$ and $\tan \delta$.

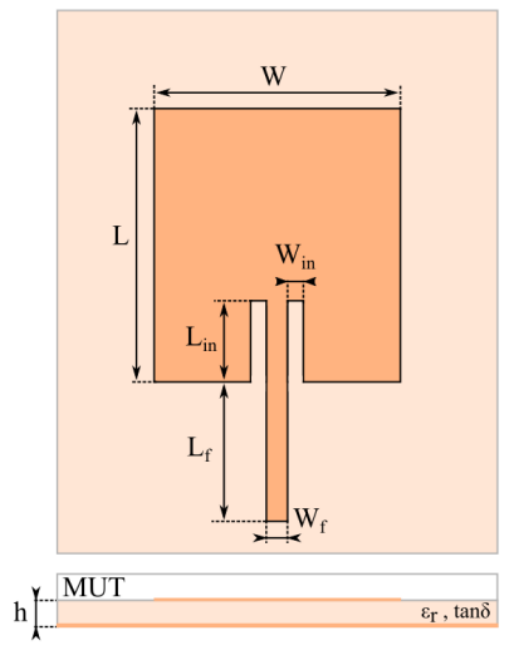

Fig. 2. Inset-fed patch antenna resonating at $2.25 \mathrm{GHz}$, with MUT on top.

TABle I. Dimensions of the Patch Antenna (SeE Fig. 2) OPERATING AT $2.25 \mathrm{GHz}$

\begin{tabular}{l|c}
\hline \hline Parameter at $2.25 \mathbf{G H z}$ & Value (mm) \\
\hline \hline Patch length $L$ & 36 \\
\hline Patch width $W$ & 36.5 \\
\hline Inset length $L_{i n}$ & 10 \\
\hline Inset width $W_{i n}$ & 3 \\
\hline Feed length $L_{f}$ & 10 \\
\hline Feed width $W_{f}$ & 1.2 \\
\hline \hline
\end{tabular}

$$
f_{\text {sim }}^{(i)}\left(x_{1}, x_{2}\right)=\sum_{k_{1}=0}^{P_{1}} \sum_{k_{2}=0}^{P_{2}} y_{k_{1} k_{2}}^{(i)} \phi_{k_{1}}\left(x_{1}\right) \phi_{k_{2}}\left(x_{2}\right)
$$

with $i=1,2 . x_{1}, x_{2} \in \Gamma=[-1,1]$ are two normalized variables corresponding to $\varepsilon_{r}$ and tan $\delta$, respectively, defined by

$$
\begin{aligned}
\varepsilon_{r}= & \left(1+x_{1}\right)\left(\frac{\varepsilon_{r}^{\text {sim }}}{2}+\Delta \varepsilon_{r}^{\text {sim }}\right) \\
& +\left(1-x_{1}\right) \frac{\max \left(\varepsilon_{r}^{\operatorname{sim}}-2 \Delta \varepsilon_{r}^{\operatorname{sim}}, 1\right)}{2} \\
\tan \delta= & \frac{\Delta \tan \delta}{2} x_{2}+\frac{\Delta \tan \delta}{2} .
\end{aligned}
$$

The functions $\phi_{k_{i}}\left(x_{i}\right)$ are orthonormal Legendre polynomials of degree $k_{i}$ corresponding to the $i$ th variable $(i=1,2)$. The total order $P$ of expansions (2) is equal to $P_{1}+P_{2}$, where $P_{1}$ and $P_{2}$ are the maximum values that can be achieved by $k_{1}$ and $k_{2}$, respectively. The coefficients $y_{k_{1} k_{2}}^{(i)}$ are calculated by applying Galerkin weighting and an $R_{1} \times R_{2}$-point GaussLegendre quadrature rule to (2), yielding 


$$
y_{k_{1} k_{2}}^{(i)} \approx \sum_{l_{1}=0}^{R_{1}} \sum_{l_{2}=0}^{R_{2}} w_{l_{1}} w_{l_{2}} \phi_{k_{1}}\left(x_{l_{1}}\right) \phi_{k_{2}}\left(x_{l_{2}}\right) f_{\mathrm{sim}}^{(i), l_{1} l_{2}}
$$

where $x_{l_{1}}$ and $x_{l_{2}}$ are the quadrature points, and $w_{l_{1}}$ and $w_{l_{2}}$ are the corresponding weights. The values $f_{\text {sim }}^{(i) l_{1} l_{2}}$ in (5) are evaluated by a full-wave electromagnetic field solver, such as Advanced Design System (ADS) Momentum. Finally, the measured values of $f_{\text {meas }}^{(1)}$ and $f_{\text {meas }}^{(2)}$, at which the measured $\left|S_{11}\right|_{\mathrm{dB}}$ crosses the threshold $t_{\mathrm{dB}}$, are compared to the approximations (2) of $f_{\mathrm{sim}}^{(1)}$ and $f_{\mathrm{sim}}^{(2)}$. The solution of the resulting system of equations yields the values of $\varepsilon_{r}$ and $\tan \delta$ of the MUT as

$$
\sum_{k_{1}=0}^{P_{1}} \sum_{k_{2}=0}^{P_{2}} y_{k_{1} k_{2}}^{(i)} \phi_{k_{1}}\left(x_{1}\right) \phi_{k_{2}}\left(x_{2}\right)=f_{\text {meas }}^{(i)} \quad i=1,2 .
$$

In case the values of $\varepsilon_{r}$ and $\tan \delta$ do not belong to the variation intervals defined at the beginning, a larger value of $\Delta \varepsilon_{r}^{\text {sim }}$ is substituted into (1) to enlarge the variation interval $\Delta \varepsilon_{r}$ and a larger interval $\Delta \tan \delta$ is considered. Then, we reconstruct the expansions (2) and reprocess the measured data until a valid solution is found. Moreover, if the MUT has already been characterized and a model is readily available, the newly measured values of $f_{\text {meas }}^{(1)}$ and $f_{\text {meas }}^{(2)}$ are directly introduced into the algorithm that determines $\varepsilon_{r}$ and $\tan \delta$. In that case, the properties of the material are immediately computed without resorting to full-wave simulations. Finally, we point out that the entire characterization process may be affected by uncertainties in the MUT thickness. To address this issue, the height $h$ of the MUT can be included as a third variable $x_{3}$ in (2), measured during the characterization process and then substituted for $x_{3}$ before solving (6). However, we found that a variation as large as $25 \%$ in $h$ results in absolute errors of less than $1 \%$ and $5 \%$ for $\varepsilon_{r}$ and $\tan \delta$, respectively. Therefore, we carry out the validation of the advocated technique by simply considering the MUT's nominal thickness.

\section{Characterization Results}

The characterization process of Section II is applied to a measurement fixture consisting of an inset-fed patch antenna (see Fig. 2), manufactured on a rigid I-Tera MT40 highfrequency laminate, with $\varepsilon_{r}=3.56, \tan \delta=0.0035$, and thickness $h=0.508 \mathrm{~mm}$. The patch is etched in a $35-\mu \mathrm{m}$-thick copper layer. The antenna has been designed in ADS momentum to resonate at $2.25 \mathrm{GHz}$, thus allowing material characterization in the proximity of the $2.45 \mathrm{GHz}$ Industrial, Scientific and Medical frequency band, which is very important for on-body communication. The design parameters of the antenna are reported in Table I. The extraction algorithm is implemented in mathematica, solving (7) in a numerical way.

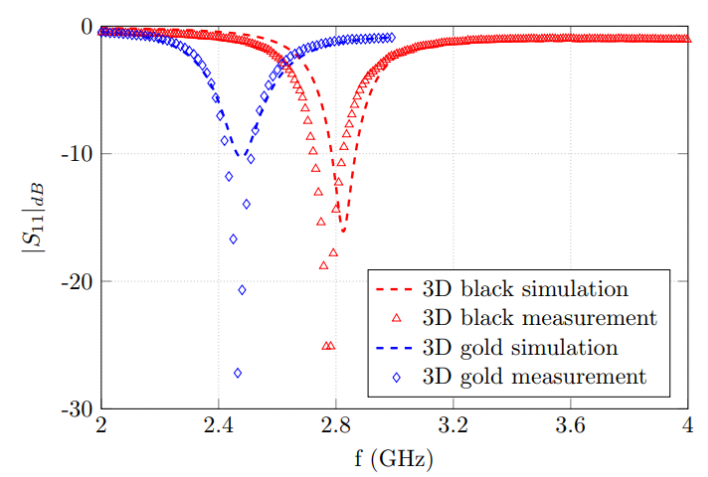

Fig. 3. Measured versus simulated $\left|S_{11}\right|_{\mathrm{dB}}$ of the two $2.45 \mathrm{GHz}$ probefed patch antennas.

TABLE II. NOMINAL CHARACTERISTICS OF THE CONSIDERED MATERIALS

\begin{tabular}{l|c|c|c}
\hline \hline \multicolumn{1}{l}{ Material } & \multicolumn{1}{c}{ Manufacturer } & Composition & h (mm) \\
\hline \hline 3-D black & Leandro Manual Arajo 3003 & $100 \%$ polyester & 2.65 \\
\hline 3-D gold & Leandro Manual Arajo 3013 & $100 \%$ polyester & 3.068 \\
\hline 3-D white & Leandro Manual Arajo 3015 & $100 \%$ polyester & 4.14 \\
\hline Corktex & Corticeira Amorim 8245 & $100 \%$ cork & 1.1 \\
\hline EVA green & - & $100 \%$ EVA & 2.01 \\
\hline Fake leather & - & $100 \%$ polyester & 0.831 \\
\hline \hline
\end{tabular}

TABLE III. Results of the Characterization Procedure

\begin{tabular}{l|c|c|c|c|c}
\hline \hline \multicolumn{1}{c}{ Material } & \multicolumn{1}{c}{ Setup Time } & $\overline{\varepsilon_{r}}$ & \multicolumn{1}{c}{$\boldsymbol{\sigma}_{\boldsymbol{\varepsilon}_{\boldsymbol{r}}}$} & \multicolumn{1}{c}{$\operatorname{\operatorname {tan}\boldsymbol {\delta }}$} & $\boldsymbol{\sigma}_{\tan \boldsymbol{\delta}}$ \\
\hline \hline 3-D black & $14 \min 52 \mathrm{~s}$ & 1.120 & $1.74 \mathrm{e}-2$ & 0.00634 & $2.36 \mathrm{e}-3$ \\
\hline 3-D gold & $14 \min 52 \mathrm{~s}$ & 1.118 & $1.70 \mathrm{e}-2$ & 0.00608 & $2.25 \mathrm{e}-3$ \\
\hline 3-D white & $14 \min 52 \mathrm{~s}$ & 1.133 & $6.16 \mathrm{e}-3$ & 0.03740 & $6.83 \mathrm{e}-4$ \\
\hline Corktex & $16 \min 13 \mathrm{~s}$ & 1.295 & $1.32 \mathrm{e}-2$ & 0.04640 & $1.07 \mathrm{e}-3$ \\
\hline EVA green & $14 \min 52 \mathrm{~s}$ & 1.103 & $1.15 \mathrm{e}-3$ & 0.03990 & $8.25 \mathrm{e}-4$ \\
\hline Fake leather & $16 \min 13 \mathrm{~s}$ & 1.266 & $4.63 \mathrm{e}-3$ & 0.04510 & $1.81 \mathrm{e}-3$ \\
\hline \hline
\end{tabular}

\section{A. Characterization of Textile Substrates}

We now characterize six substrates of potential interest for antenna production. Their normal characteristics are reported in Table II. In particular, 3-D fabrics are applied in the manufacturing of protective clothing, e.g., for firefighters, whereas fake leather and EVA substrates are popular in fashion design and sport clothing, respectively. In contrast, cork is becoming more and more important in the production of components for cars and other vehicles.

A sample of each material was placed on top of the patch antenna, and the corresponding reflection loss $\left|S_{11}\right|_{\mathrm{dB}}$ was measured. Then, for each new material, the characterization procedure of Section II was carried out. Since the materials were expected to exhibit a low permittivity, $\varepsilon_{r}^{\text {sim }}$ was set to 1 , whereas $\Delta \varepsilon_{r}^{\text {sim }}$ was kept equal to 0.1 . The loss tangent $\tan \delta^{\text {sim }}$ was set to the mean value of the variation interval $\Delta \tan \delta$, being 0.05 . The threshold $t_{\mathrm{dB}}$ was kept equal to $-8 \mathrm{~dB}$ for all substrates. All the models converged with orders of expansion $P_{1}=P_{2}=2$. 
For each material, a square sample was measured by positioning it such that each time one different side is aligned with the microstrip feed of the antenna and by repeating the procedure by turning it upside down. The mean value and the variance of the results for both the permittivity and the loss tangent, as well as the setup time, are reported in Table III. For each material, the extraction of all eight results took $1.28 \mathrm{~s}$.

As a final validation two prototypes of a probe-fed textile patch antenna were designed with CST Microwave Studio to resonate between 2 and $3 \mathrm{GHz}$. They were manufactured with the 3-D black and the 3-D gold substrates. A commercial etextile with resistivity $\rho_{s}=0.18 \Omega / \mathrm{sq}$ and thickness $80 \mu \mathrm{m}$ was applied for both the ground plane and the patch. The first antenna consist of a 45.37-mm-long and 35-mm-wide rectangular patch, with the feed positioned at $8.5 \mathrm{~mm}$ from the center toward the short edge of the patch. In contrast, the second prototype exhibits a square patch length of $52.5 \mathrm{~mm}$ and feed at $15 \mathrm{~mm}$ from the center of the patch. Both measured and simulated $\left|S_{11}\right|_{\mathrm{dB}}$ of both antennas are shown in Fig. 3. We notice that a very good agreement between measurements and simulations is found for the prototype manufactured with the 3D gold substrate. The measured resonance peak of the prototype manufactured with the 3-D black material is slightly shifted with respect to the simulated one. Such a shift corresponds to a difference in the material's $\varepsilon_{r}$ as small as 0.04 with respect to the value obtained by the characterization process. This we ascribe to the presence of the glue applied to attach both the patch and the ground plane to the substrate [10]. Such an effect may also be accounted for during antenna fabrication.

\section{CONCLUSION}

A novel technique was proposed for the characterization of the electromagnetic properties of substrate materials for textile antenna design. This non-destructive method is based on an inset-fed patch antenna interacting with an MUT. The frequency values for which the resonance peak of the return loss $\left|S_{11}\right|_{\mathrm{dB}}$ crossed a given threshold value are modeled as functions of the relative permittivity $\varepsilon_{r}$ and the loss tangent $\tan \delta$. The comparison between these functions and the measured data yield the electromagnetic properties of the material under test. The proposed approach has proven to be fast, accurate, and flexible. Therefore, it is compatible with an assembly-line fabrication, thus considerably improving the efficiency of the production of textile antennas.

\section{REFERENCES}

[1] J. Lilja et al., "Body-worn antennas making a splash: Lifejacket-integrated antennas for global search and rescue satellite system," IEEE Antennas Propag. Mag., vol. 55, no. 2, pp. 324-341, Apr. 2013.

[2] T. Castel et al., "Capacity of broadband body-to-body channels between firefighters wearing textile SIW antennas," IEEE Trans. Antennas Propag., vol. 64, no. 5, pp. 1918-1931, May 2016.

[3] P. Salonen, Y. Rahmat-Samii, H. Hurme, and M. Kivikoski, "Effect of conductive material on wearable antenna performance: A case study of WLAN antennas," in Proc. IEEE Antennas Propag. Soc. Int. Symp., Jun. 2004, vol. 1, pp. 455-458.

[4] P. Salonen, Y. Rahmat-Samii, M. Schaffrath, and M. Kivikoski, "Effect of textile materials on wearable antenna performance: A case study of GPS antennas," in Proc. IEEE Antennas Propag. Soc. Int. Symp, 2004, vol. 1, pp. 459-462.

[5] D. Cottet, J. Grzyb, T. Kirstein, and G. Troster," "Electrical characterization of textile transmission lines," IEEE Trans. Adv. Packag., vol. 26, no. 2, pp. 182-190, May 2003.

[6] L. F. Chen, C. K. Ong, C. P. Neo, V. V. Varadan, and V. K. Varadan, Microwave Electronics: Measurement and Materials Characterization. New York, NY, USA: Wiley, 2004.

[7] C. Hertleer, A. Van Laere, H. Rogier, and L. Van Langenhove, "Influence of relative humidity on textile antenna performance," Textile Res. J., vol. 80, pp. 177-183, Jan. 2010.

[8] M. Rossi, S. Agneessens, H. Rogier, and D. Vande Ginste, "Stochas-tic analysis of the impact of substrate compression on the performance of textile antennas," IEEE Trans. Antennas Propag., vol. 64, no. 6, pp. 2507-2512, Jun. 2016.

[9] Y. Ouyang and W. J. Chappell, "High frequency properties of electrotextiles for wearable antenna applications," IEEE Trans. Antennas Propag., vol. 56, no. 2, pp. 381-389, Feb. 2008.

[10] F. Declercq, H. Rogier, and C. Hertleer, "Permittivity and loss tan-gent characterization for garment antennas based on a new matrix-pencil twoline method," IEEE Trans. Antennas Propag., vol. 56, no. 8, pp. 2548-2554, Aug. 2008.

[11] F. Declercq, I. Couckuyt, H. Rogier, and T. Dhaene, "Environmental high frequency characterization of fabrics based on a novel surrogate mod-elling antenna technique," IEEE Trans. Antennas Propag., vol. 61, no. 10, pp. 5200-5213, Oct. 2013. 